\title{
Video Shadow Positioning Technology
}

\author{
Zhicheng Huang
}

School of North China Electric Power University, Baoding 071000, China;

648277489@qq.com

Keywords: solar elevation angle, grid search technique, Quasi-Newton iterative method, video imaging principle

\begin{abstract}
This paper mainly studies the technology of accurately positioning the imaging position through the changes of shadow length in video. 3D modeling software SketchUp is applied to conduct hidden line processing on images and obtain the actual shadow length of objects in the video. Take into account the impact of various factors, a precise model that the shadow length changes over time is established; on this basis, a model that reversely positioning the shadow position is also constructed, with the purpose of maintain the minimum sum between object height variation and rotation angle error, besides, latitude and longitude are regarded as the independent variables. As the function relation of parameters is both complex and non-linear, so grid method is firstly adopted, to obtain the approximate location of possible measuring areas, and then based on the initial value to obtain a corresponding precise position by quasi-Newton iterative method, finally obtain the actual shooting location of objects.
\end{abstract}

\section{Introduction}

The study of shadow has a long history; in ancient China, sundial was even invented based on the changes of shadow. Nowadays, some scholars have carried out a large number of studies on the technology of shadow positioning, but the considered factors are not comprehensive, and the errors are large. For example, literature [1] did not consider corrections on apparent, mean solar time and refractive index, so the error was quite large. Currently, depth research has been done on solar elevation angle, rotation angle and atmospheric refraction index both in domestic and foreign countries. This paper proposed an accurate shadow length change model based on the research on atmospheric refraction index in literature [2] and research on solar azimuth in literature [3]. Furthermore, the technology that reversely solves video shooting position is also obtained with grid search technique and Quasi-Newton iterative method.

\section{Shadow length changes model over time}

The height of object is known, as long as the solar elevation angle is obtained, you can get the length of the shadow, the formula is as follows:

$\mathrm{L}=\mathrm{H} * \cot h$

Wherein, $\mathrm{L}$ is the length of the shadow, $\mathrm{H}$ is the object height, and $\mathrm{h}$ is the solar elevation angle. By reviewing relevant geographic data, the calculation formula ${ }^{[1]}$ of solar elevation angle can be obtained:

$\sin h=\sin \delta \sin \varphi+\cos \delta \cos \varphi \cos \tau$

Wherein, $\delta$ is the angle of declination, $\varphi$ is the latitude of the location, and $\tau$ is the hour angle of a certain time.

Declination angle is calculated:

$$
\delta=23.45^{\circ} * \sin \left(\frac{2 \pi(284+N)}{365}\right)
$$

Wherein, $\mathrm{N}$ is the date serial number (accumulative day), i.e. consider New Year's Day as a benchmark (the accumulative day of January 1 is 1), the order number of date in a year.

In the 24-hour format, hour angle is calculated: 
$\tau=15^{\circ} *(\mathrm{t}-12)$

Wherein, $\mathrm{t}$ is the local time of time to be resolved, take Beijing as an example, its conversion relation is:

$\mathrm{t}=t_{\text {Beijing }}+t_{\Delta}$

$t_{\text {Beijing }}$ is Beijing time (Eastern eight zone), $\mathrm{t}_{\Delta}$ is the time difference, time difference is the ratio between longitude difference between east longitude $120^{\circ}$ and the location and $15^{\circ}$ (in hour).

\subsection{Correction on model parameters}

1) Time correction

Time difference exists between apparent solar time and mean solar time, and it changes over date, but not related to location. In daily life, the time we used is mean solar time, but the time that involved in solar elevation angle and hour angle calculation formula is apparent solar time, so time correction is required. We can obtain the time difference $E_{t}$.

Thus, the relationship between apparent solar time and mean solar time is:

$S_{\text {true sun }}=S_{\text {ping sun }}+E_{t}$

2) Correction on solar elevation angle

As the atmosphere and the air have refraction on sunlight. It is ideal solar elevation angle in the calculation formula used in this paper, but the refracted sunlight refraction angle becomes small, and thus the actual solar elevation angle is larger than the ideal elevation angle obtained from formula calculation, so the solar elevation angle needs to be corrected.

Atmospheric refractivity [2]:

$n_{t p}=1+\frac{p\left(n_{s}-1\right)}{96095.43} * \frac{1+10^{-8}(0.613-0.00998 t) p}{1+0.0036610 t}$

Of which, $n_{s}$ refers to the refractivity under the condition of a standard atmospheric pressure, $15{ }^{\circ} \mathrm{C}$, $\mathrm{CO}_{2}$ equals to $300 \mathrm{ppm}$ in standard air $(78.09 \%$ nitrogen, $20.93 \%, 0.93 \%$ argon, $0.03 \%$ carbon dioxide).

$n_{s}=1+\left(83.4213+\frac{24060.3}{130-\sigma^{2}}+\frac{159.97}{41-\sigma^{2}}\right) \times 10^{-6}$

Wherein, $\sigma$ is the wave number, with the unit $\mu \mathrm{m}^{-1}$, taking the wavelength of $700 \mathrm{~nm}$.

Assume the angle of emergence after refraction to be $\psi$, and the following can be obtained with geometric analysis:

$$
\begin{aligned}
& \sin \psi=\frac{\sin \left(90^{\circ}-h\right)}{n} \\
& h^{\prime}=90^{\circ}-\psi
\end{aligned}
$$

Among them, $h$ is the sun elevation angle after correction.

NOTE: All formulas in this paper, east longitude is positive, and west longitude is negative, north latitude is positive, and south latitude is negative. All parameters that require correction and formulas require improvement that involved in 1.1 are adopted in formulas (1) to (5).

\section{Shadow Length Reversed Positioning Model}

Shadow changes include shadow length and movement angle velocity two aspects. When the object height is unknown, using grid method for solution and by determining the latitude and longitude of grid to determine the solar elevation angle in grid representative area, so as to obtain object height through shadow length, minimum height change is regarded as a measurement index; moreover, taking into account that even several possible measurement places with constant object height can be obtained by grid method, but due to different latitudes, the rotation angular velocity of shadow will also be different. The solar azimuth can be considered approximately as the angle between the shadow of erected object on the ground under the sun and direct south, so we select the solar azimuth differences of each grid adjacent moment and shadow moving angle, the minimum difference between the two is regarded as another measurement index. At this time, the sum of two 
indicators is regarded as the objective function, so the problem is converted to a single objective programming model. The approximate location of several possible places can be solved by using MATLAB, and then use the quasi-Newton iterative method for obtaining a more accurate measurement places. Solution process is as follows:

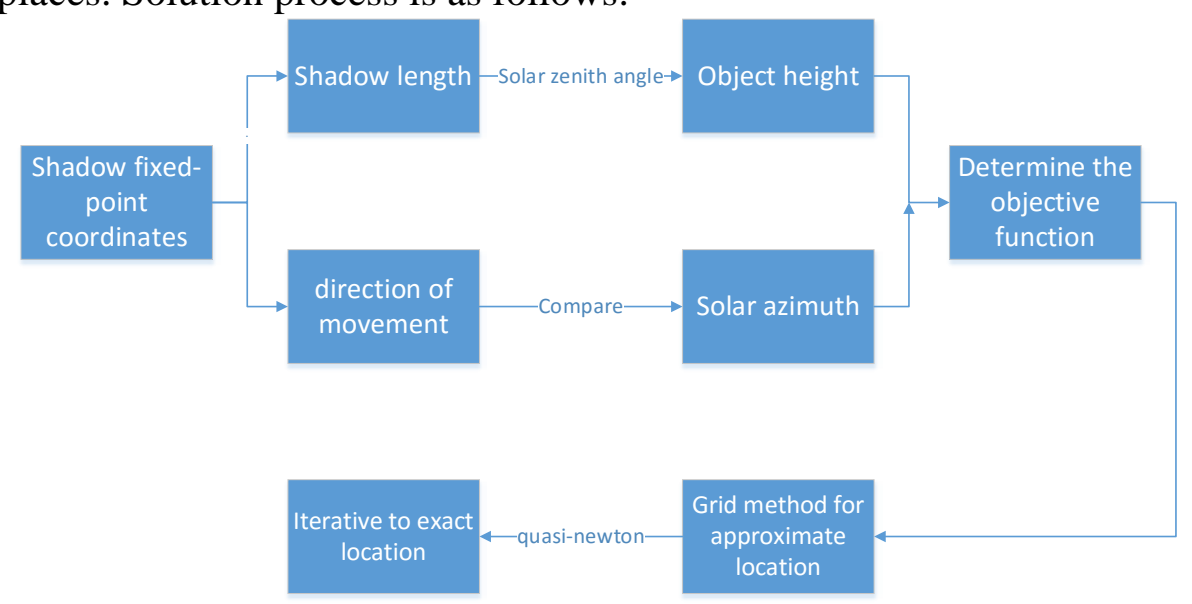

Fig.1 Model 2 application flow chart

\subsection{Resolve the approximate location of possible places with grid method}

Firstly, $1^{\circ} \times 1^{\circ}$ spatial resolution is adopted to divide the globe into a plurality of uniform grids, thus the latitude and longitude of each grid can be determined, and then use equation (1), (2), (4), (5), (7) to obtain the solar elevation angle $h_{i}$ and objects height $H_{i}$ of each measurement hour for each grid on April 18, 2015, the solar azimuth $A_{i}$ can also be obtained, solar azimuth is calculated as follows [1]:

$$
\cos \mathrm{A}=(\sinh \sin \varphi-\sin \delta) / \cosh \cos \varphi
$$

Take the shadow relative rotational angle of two adjacent measuring times $\alpha$, use the resolved solar azimuth of each measuring time to obtain the solar azimuth time difference $\Delta A_{i}$ of adjacent times, in this paper, $\Delta A_{i}$ is known as the rotation angle of solar azimuth, solar azimuth changes and shadow movement angle at the same time represent the sun azimuth changes speed and shadow movement angle velocity, respectively, hence two metrics are obtained as follows:

$$
\begin{aligned}
& \min \sum_{i=0}^{21}\left|H_{i}-\bar{H}\right| \\
& \min \sum_{i=1}^{20}\left|\Delta A_{i}-\alpha_{i}\right|
\end{aligned}
$$

The two indicators are added to obtain an objective function, and a single objective programming model is established:

$$
\begin{gathered}
\min \left(\sum_{i=0}^{21}\left|H_{i}-\bar{H}\right|+\sum_{i=1}^{20}\left|\Delta A_{i}-\alpha_{i}\right|\right) \\
\text { s.t. }\left\{\begin{array}{c}
-90^{\circ} \leq \varphi \leq 90^{\circ} \\
-180^{\circ} \leq \phi \leq 180 \\
1 \leq N \leq 365, N \text { is the integer }
\end{array}\right.
\end{gathered}
$$

Wherein, $\bar{H}$ is the averaged object height calculated from all measuring hours.

$$
\bar{H}=\frac{1}{n} \sum_{i=1}^{n} H_{i}
$$

Since the spatial resolution adopted by grid method is $1^{\circ} \times 1^{\circ}$, so the latitude and longitude in solution result have lower accuracy, and the obtained initial result is quite inaccurate. In this way, the approximate locations of two possible measurement places are regarded as the initial value, by calling 
MATLAB optimization toolbox to have Quasi-Newton iterative, and obtain the exact solution of measurement places.

\subsection{Model Inspection}

In this paper, related data inspection model provided by 2015 Mathematical Modeling Contest of China Higher Education Press Cup is applied.

Using the above model, and MATLAB to resolve the distribution diagram of objective function for each grid as follows:

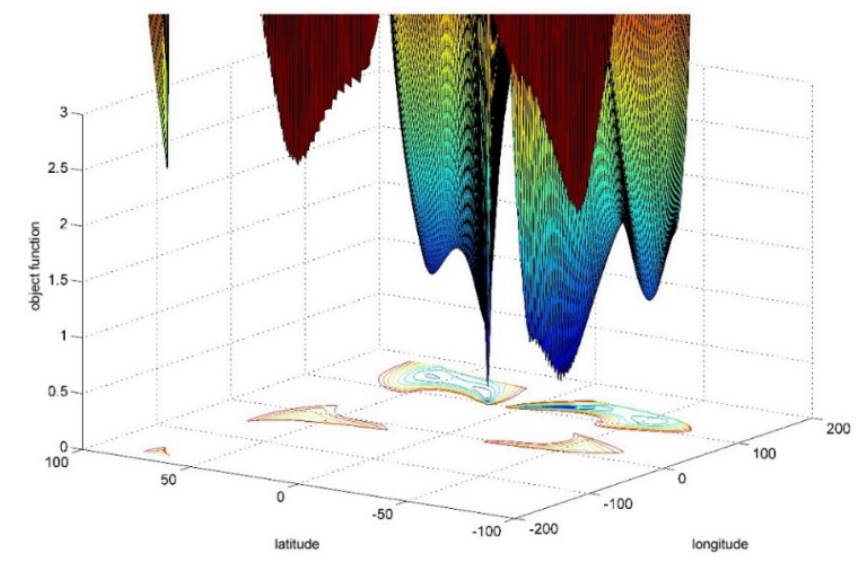

Fig.2 Distribution diagram of grid method objective function

\section{Get the actual shadow length from video}

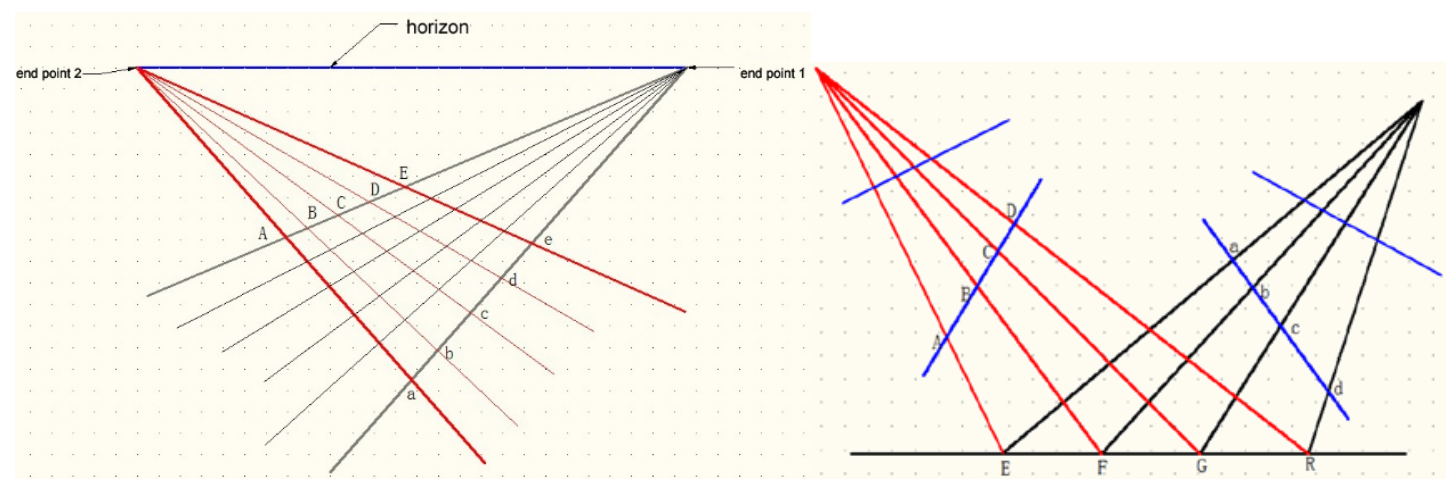

Fig.3 Schematic diagram of imaging property 1 and property 2

Imaging property 1: In Fig. 12, Aa, Bb, Cc, Dd, Ee are paired parallel lines in three-dimensional space; similarly, EA also paralleled to ea. Parallel straight lines are mapped to intersecting straight line in Figure 13 through the camera, this intersection is called as vanishing point. Two sets of intersecting parallel lines form one plane and two vanishing points, and the connection of the two vanishing points is horizon. Therefore, the key of observing data in image is to find the vanishing points.

Imaging property 2: The camera projected three-dimensional space onto a two-dimensional image to ensure the cross ratio of straight line remains unchanged. As shown above, there are EF / EG $=\mathrm{AB}$ / $\mathrm{AC}=\mathrm{ab} / \mathrm{ac}, \mathrm{FG} / \mathrm{GR}=\mathrm{BC} / \mathrm{CD}=\mathrm{bc} / \mathrm{cd}$.

By taking advantage of these two properties and referring to club length, reliable shadow length can be obtained under the established local dimensional coordinate system in SketchUp, and then the shooting position can be resolved in next step.

\section{Conclusion}

In this paper, it fully considered the impact of atmospheric refraction angle, apparent/ mean solar time and time zone difference on shadow length changes, and accurate model of shadow length varies over time is obtained. Through video imaging principle, a local three-dimensional coordinate system 
is established with SketchUp software, and with the purpose to resolve the actual shadow length in video. In the following, use the model of shadow length varies over time and the accurate shadow length extracts from video, to obtain the initial value of shooting location with grid search technique, and then obtain the accurate shooting position with quasi-Newton iterative method. However, the model established in this paper applies only to the situation when the shooting time is known, and further studies still required if the shooting time is unknown.

\section{Reference}

[1]. Wang Guo'an1,Mi Hongtao2,Deng Tianhong2,Li Ya'nan3,et al. Calculation of the annual variation range of the solar azimuth and the sun azimuth angle at sunrise and sunset time. Meteorology and Environmental Science .Vol.30 (2007). Suppl

[2].Jin Fengqun:Study on factors affecting atmospheric refractive index(Master, Zhejiang University, china, 2006), p9-12.

[3]. He Xiaolei ,Yu Hejun Li, Jianying Ding Lei. Formula solution of solar azimuth and its application .Acta Energiae Solaris Sinica. Vol.29(2008),No.1

[4] information on : http : // wenku. baidu. com / link ? url = Ui S xE idU ay -sf o2 ZZ 6E I Iw U4K J eO 1 u m D - Q o 3G lu D I P g w 7 u W 1 w a a p r x2 7 q W c r 6 s r q M b Y O I j x iZ W p O c O o -8 Ya Z H p 7 I u L s w c b A x 9J _ 4 nA7 xa AK 